\title{
Accuracy and Efficiency Considerations in the Solution of Extremely Large Electromagnetics Problems
}

\author{
Özgür Ergül ${ }^{1}$ and Levent Gürel ${ }^{2,3}$ \\ ${ }^{1}$ Department of Mathematics and Statistics \\ University of Strathclyde, G11XH, Glasgow, UK \\ ${ }^{2}$ Department of Electrical and Electronics Engineering \\ ${ }^{3}$ Computational Electromagnetics Research Center (BiLCEM) \\ Bilkent University, TR-06800, Bilkent, Ankara, Turkey \\ ozgur.ergul@strath.ac.uk, lgurel@bilkent.edu.tr
}

\begin{abstract}
Fast and accurate solutions of extremely large electromagnetics problems are considered. Surface formulations of large-scale objects lead to dense matrix equations involving millions of unknowns. Thanks to the recent developments in parallel algorithms and high-performance computers, these problems can easily be solved with unprecedented levels of accuracy and detail. For example, using a parallel implementation of the multilevel fast multipole algorithm (MLFMA), we are able to solve electromagnetics problems discretized with hundreds of millions of unknowns. Unfortunately, as the problem size grows, it becomes difficult to assess the accuracy and efficiency of the solutions, especially for the comparison of different implementations. This paper presents our efforts on the solution of extremely large electromagnetics problems with the emphasis on the accuracy and efficiency. We present a list of benchmark problems, which can be used to compare different implementations for large-scale problems.
\end{abstract}

\section{INTRODUCTION}

Real-life electromagnetics problems often involve very large objects with respect to wavelength. Accurate formulations of these problems with the surface integral equations lead to dense matrix equations involving millions of unknowns. Recently, there has been many efforts to increase the problem size from millions to hundreds of millions using highperformance computing techniques on parallel computers [1][14]. Specifically, parallelization of the fast algorithms, such as the multilevel fast multipole algorithm (MLFMA) [15], has enabled the solution of extremely large electromagnetics problems, discretized as many as one billion unknowns [16]. At the same time, with the rapid increase in the number of unknowns, it becomes crucial but more difficult to assess the accuracy and efficiency of the algorithms and their parallel implementations [17].

This paper presents our efforts for rigorous solutions of extremely large electromagnetics problems. We discuss the assessment of the accuracy and efficiency of parallel algorithms and their implementations. Based on these discussions, we present a set of benchmark problems involving canonical objects to test and compare different solvers for large-scale problems. We also present the solution of these benchmark problems using a sophisticated parallel implementation of MLFMA. Details of this implementation can be found in [9] and [14].

\section{EFFICIENCY CONSIDERATIONS}

Measuring the efficiency of a parallel solution is not trivial. Parallelization speedup and efficiency results, which are often used to demonstrate the effectiveness of the parallel implementations, can be misleading [9]. A major problem is that the parallelization speedup is usually measured as the reduction in the processing time with respect to a single-processor solution, but it does not give any information on the processing time on a single processor, i.e., the efficiency of the algorithm itself. Specifically, a very slow algorithm can be "embarrassingly" parallelizable leading to very high parallelization speedup and efficiency, but this does not mean that the implementation and solutions are efficient. Unfortunately, a very common practice in the literature is to increase the parallelization speedup and efficiency by increasing the processing time, e.g., by performing some of the computations on the fly and parallelizing them very efficiently. Obviously, these implementations may not be as efficient as claimed and the related efficiency results are often exaggerated.

Another disadvantage of using the parallelization speedup and efficiency is their complicated dependence to the parallel computer and architecture. For example, using slower processors without changing the communication network may increase the parallelization efficiency. This is because the computations, which take longer on the slower processors, dominate the communications. Having relatively short communication time in the overall processing time translates into higher parallelization speedup and efficiency. But, again, the actual efficiency of the solution can be very low due to the slower processors.

Considering the disadvantages of using the parallelization speedup and efficiency, the processing time itself can be used 
to measure the actual efficiency of the parallel implementations. The processing time also depends on the parallel computer. But, computer specifications and solution parameters, such as the number of processors, distribution of processes, processor models, clock rates, and the network speed can be provided along with the processing time. This may lead to more fair comparisons of the parallel implementations. Solutions of multiple problems with different sizes can increase the reliability of the comparisons.

\section{ACCURACY CONSIDERATIONS}

Accuracy is easier to assess but often discarded in largescale computations [17]. There are many error sources in numerical solutions, such as the discretization of the geometry and equations, numerical integration, factorization, diagonalization, interpolation, and iterative convergence. All these error sources must be suppressed to obtain accurate results. Accuracy of solutions must accompany the efficiency results. For example, reducing the accuracy may reduce the processing time and increase the parallelization efficiency. Unfortunately, decreasing the number of integration points (in numerical integrations), decreasing the number of harmonics (in factorizations), decreasing the number of samples (in interpolations), and increasing the target residual error (in iterative solutions) are commonly practiced in the literature. Some of these relaxations may have significant effects in the final solutions. Hence, without indicating the accuracy, the efficiency results can be again exaggerated and misleading to assess the effectiveness of the implementations.

\section{BENCHMARK PROBLEMS}

In order to facilitate the assessment of parallel implementations and for their fair comparisons, we determined a set of benchmark problems involving two canonical objects, namely, the sphere and NASA Almond [19]. The sphere has a radius of $0.3 \mathrm{~m}$ and it is investigated at 11 different frequencies from $20 \mathrm{GHz}$ to $340 \mathrm{GHz}$. The NASA Almond has a size of $0.252474 \mathrm{~m}$ and it is investigated at 5 different frequencies from $112.5 \mathrm{GHz}$ to $1.8 \mathrm{THz}$. Both objects are illuminated by plane waves. For the NASA Almond, two different illuminations are considered; headon illumination and $30^{\circ}$ illumination. In both cases, the electric field is polarized horizontally. We provide the reference solutions of these problems in an interactive web site at wWw.cem.bilkent.edu.tr/benchmark. Computational values for the far-zone electromagnetic fields can be uploaded in this site. For the sphere, the uploaded results are compared to the analytical Mie-series solutions (obtained with $10^{-6}$ error). For the NASA Almond, comparisons are made against our numerical solutions (obtained with $10^{-2}$ error). In all cases, relative errors are calculated to assess the accuracy of the numerical solutions. In the next section, we present examples to the solutions of the benchmark problems performed by our parallel implementation of MLFMA.
TABLE I

Solutions of Scattering Problems Involving a SPHERE of RADIUS 0.3 M AT VARIOUS FREQUENCIES

\begin{tabular}{|c|c|c|c|c|}
\hline Diameter & Unknowns & $\begin{array}{c}\text { Iterations } \\
\left(10^{-3} \text { Residual }\right)\end{array}$ & $\begin{array}{c}\text { Total Time } \\
(\text { minutes })\end{array}$ & $\begin{array}{c}\text { Error } \\
\text { in RCS }\end{array}$ \\
\hline $40.0 \lambda$ & $1,462,854$ & 21 & 4 & 0.0092 \\
\hline $80.1 \lambda$ & $5,851,416$ & 27 & 16 & 0.0097 \\
\hline $160.1 \lambda$ & $23,405,664$ & 33 & 61 & 0.0099 \\
\hline $192.1 \lambda$ & $33,791,232$ & 39 & 107 & 0.0098 \\
\hline $240.2 \lambda$ & $53,112,384$ & 44 & 183 & 0.0099 \\
\hline $320.2 \lambda$ & $93,622,656$ & 47 & 333 & 0.0104 \\
\hline $360.3 \lambda$ & $135,164,928$ & 47 & 471 & 0.0093 \\
\hline $420.3 \lambda$ & $204,823,296$ & 50 & 647 & 0.0071 \\
\hline $520.4 \lambda$ & $307,531,008$ & 55 & 1080 & 0.0089 \\
\hline $560.4 \lambda$ & $374,490,624$ & 58 & 1430 & 0.0085 \\
\hline $680.5 \lambda$ & $540,659,712$ & 65 & $3632^{*}$ & 0.0084 \\
\hline$*$ Parallelized into 64 processes. \\
\hline \multicolumn{5}{|l}{} \\
\hline
\end{tabular}
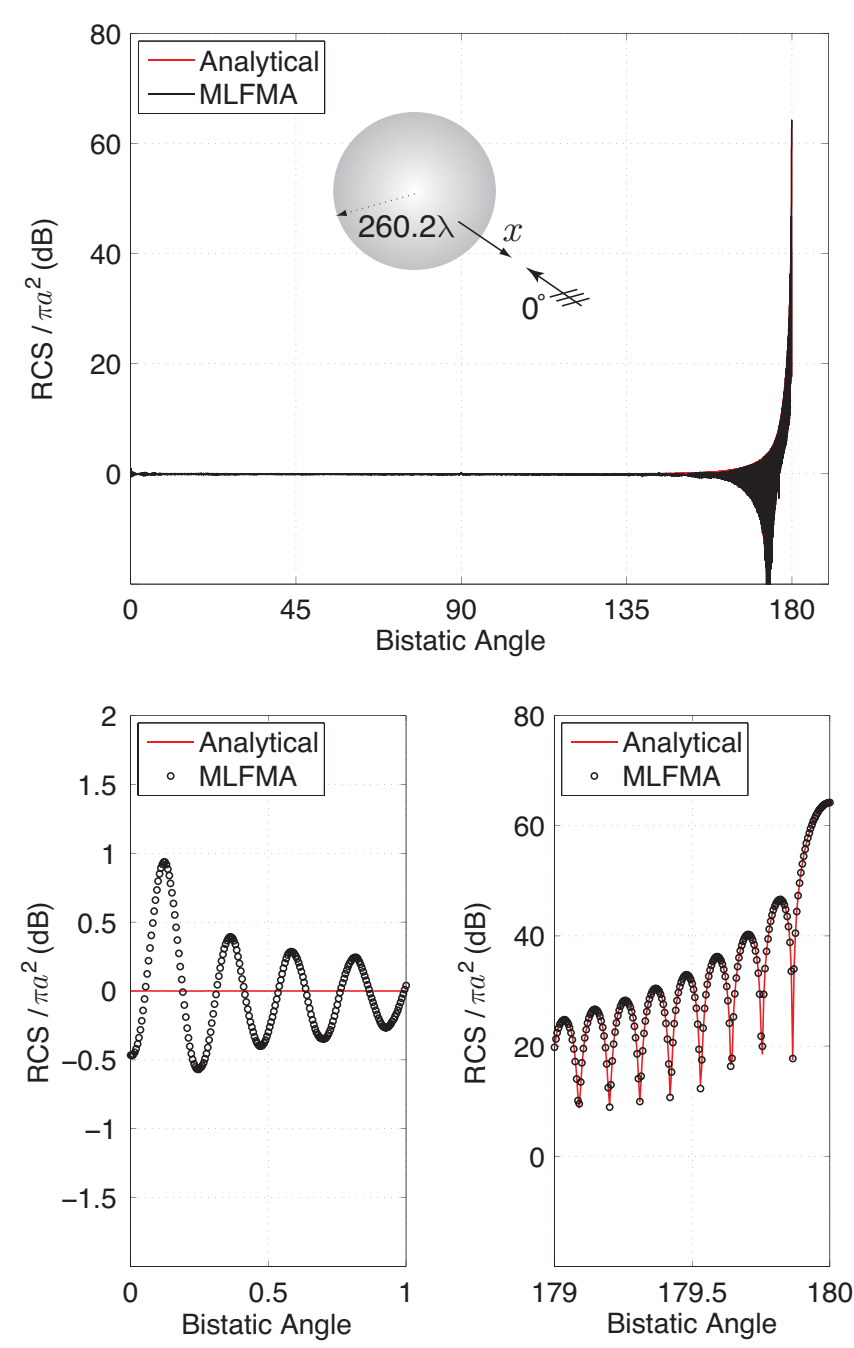

Fig. 1. Normalized bistatic RCS $\left(\mathrm{RCS} / \pi a^{2}\right)$ of a sphere with a radius of $340 \lambda$ from $0^{\circ}$ to $180^{\circ}$, where $0^{\circ}$ and $180^{\circ}$ correspond to the back-scattering and forward-scattering directions, respectively. RCS values are zoomed around the back-scattering and forward-scattering directions in separate plots.

\section{Numerical Results}

For numerical solutions, we use a parallel implementation of MLFMA based on the hierarchical partitioning strategy [9]. 
All problems are formulated with the combined-field integral equation [20] using 0.5 combination parameter and discretized with the Rao-Wilton-Glisson functions [21] on $\lambda / 10$ triangles, where $\lambda$ is the wavelength. Matrix elements are calculated with maximum $1 \%$ error. Interpolations and anterpolations are performed locally by using $6 \times 6$ stencils. Iterative solutions are performed by using the biconjugategradient-stabilized (BiCGStab) algorithm [22] and iterations are carried out until the residual error is reduced to below $10^{-3}$. With these parameters, the target error in the far-zone electromagnetic fields is $1 \%$.

Table I presents the solution of the sphere problems on a cluster of Intel Xeon Nehalem-EX L7555 processors with $1.87 \mathrm{GHz}$ clock rate. All solutions are parallelized into 128 processes, except the solution of the largest problem that is parallelized into 64 processes. Processes are distributed among 16 computing nodes. The diameter of the sphere changes approximately from $40 \lambda$ to $540 \lambda$, whereas the corresponding number of unknowns changes from $1,462,854$ to $540,659,712$. Table I lists the number of iterations, the total computing time in minutes, and the error in the far-zone electromagnetic fields. Note that the smallest and largest problems are solved in about 4 minutes and 60 hours, respectively. The error in the far-zone fields is generally less than $1 \%$, confirming that the desired accuracy is satisfied for these large-scale problems. Fig. 1 depicts the bistatic radar cross section (RCS) of the sphere at $340 \mathrm{GHz}$. It can be observed that the computational values agree very well with the analytical results obtained by using a Mie-series solution.

Table II presents the solution of the NASA Almond problems again on a cluster of Intel Xeon Nehalem-EX L7555 processors with $1.87 \mathrm{GHz}$ clock rate. All solutions are parallelized into 64 processes distributed among 16 computing nodes. The size of the NASA Almond changes approximately from $94.7 \lambda$ to $1515.3 \lambda$. The smallest and largest problems are discretized with $2,157,462$ and 552,310,272 unknowns, respectively. Table II lists the number of iterations and the total computing time for two different illumination angles. Fig. 2 depicts the bistatic RCS of the NASA Almond at $1.8 \mathrm{THz}$ when it is illuminated by a plane wave propagating towards its nose (head-on illumination). Since the RCS values are obtained with maximum $1 \%$ error, Fig. 2 can be used as a reference for high-frequency techniques.

In addition to the canonical problems involving the sphere and NASA Almond, we also employ our parallel implementation to solve more complicated problems. As an example, Fig. 3 presents the solution of a scattering problems involving the stealth airborne target Flamme [23]. The scaled size of the target is $0.6 \mathrm{~m}$ and it is investigated at $820 \mathrm{GHz}$, i.e., when its size is approximately $1641.1 \lambda$. Fig. 3 depicts the bistatic RCS of the target for the head-on illumination. As opposed to the RCS of the NASA Almond, the RCS of the Flamme exhibits specular reflections and the cross-polar component is significantly large.
TABLE II

Solutions of ScAtTering Problems Involving the NASA Almond AT VARIOUS FREQUENCIES

\begin{tabular}{|c|c|c|c|c|c|}
\hline Size & Unknowns & \multicolumn{2}{|c|}{$\begin{array}{c}\text { Iterations } \\
\left(10^{-3} \text { Residual }\right)\end{array}$} & \multicolumn{2}{c|}{$\begin{array}{c}\text { Total Time } \\
\text { (minutes) }\end{array}$} \\
\hline & & $0^{\circ}$ & $30^{\circ}$ & $0^{\circ}$ & $30^{\circ}$ \\
\hline $94.7 \lambda$ & $2,157,462$ & 27 & 26 & 8 & 8 \\
\hline $189.4 \lambda$ & $8,629,848$ & 31 & 27 & 33 & 31 \\
\hline $378.8 \lambda$ & $34,519,392$ & 40 & 32 & 148 & 134 \\
\hline $757.7 \lambda$ & $138,077,568$ & 47 & 50 & 634 & 654 \\
\hline $1515.3 \lambda$ & $552,310,272$ & 68 & 70 & 3269 & 3396 \\
\hline
\end{tabular}

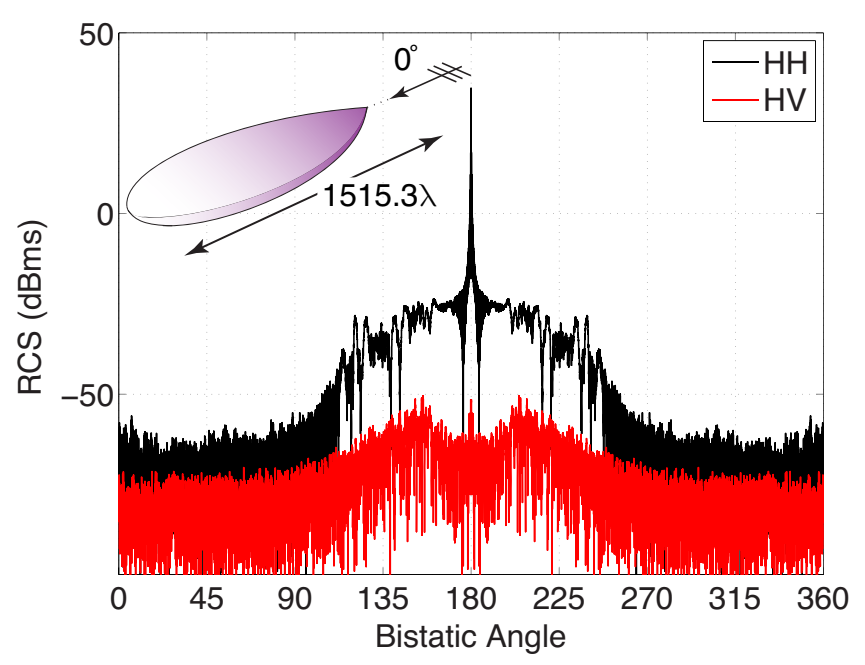

Fig. 2. Co-polar (HH) and cross-polar (HV) bistatic RCS (dBms) of the NASA Almond at $1.8 \mathrm{THz}$. The target is illuminated by a plane wave propagating towards its nose with the electric field polarized horizontally.

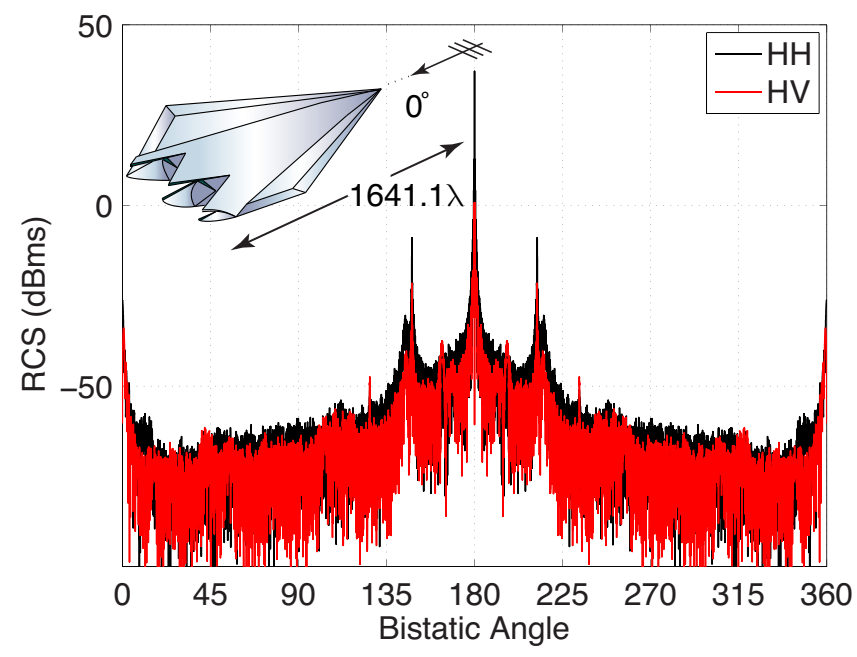

Fig. 3. Co-polar (HH) and cross-polar (HV) bistatic RCS (dBms) of the Flamme at $820 \mathrm{GHz}$. The target is illuminated by a plane wave propagating towards its nose with the electric field polarized horizontally.

\section{CONCLUSIONS}

It is extremely important to precisely assess the accuracy and efficiency of the parallel implementations for large-scale electromagnetics problems. In this study, we present a set of benchmark problems, which can be used to compare 
different implementations. We also present rigorous solutions of these problems discretized with millions of unknowns using a parallel implementation of MLFMA. Reference solutions are also available in an interactive web site at www. cem.bilkent. edu.tr/benchmark.

\section{ACKNOWLEDGMENT}

This work was supported by the Scientific and Technical Research Council of Turkey (TUBITAK) under Research Grant 110E268, by the Centre for Numerical Algorithms and Intelligent Software (EPSRC-EP/G036136/1), and by contracts from ASELSAN and SSM.

\section{REFERENCES}

[1] S. Velamparambil, W. C. Chew, and J. Song, "10 million unknowns: Is it that big?," IEEE Antennas Propag. Mag., vol. 45, no. 2, pp. 43-58, Apr. 2003.

[2] L. Gürel and Ö. Ergül, "Fast and accurate solutions of integral-equation formulations discretised with tens of millions of unknowns," Electron. Lett., vol. 43, no. 9, pp. 499-500, Apr. 2007.

[3] Ö. Ergül and L. Gürel, "Hierarchical parallelisation strategy for multilevel fast multipole algorithm in computational electromagnetics," Electron. Lett., vol. 44, no. 1, pp. 3-5, Jan. 2008.

[4] X.-M. Pan and X.-Q. Sheng, "A sophisticated parallel MLFMA for scattering by extremely large targets," IEEE Antennas Propag. Mag., vol. 50, no. 3, pp. 129-138, Jun. 2008.

[5] Ö. Ergül and L. Gürel, "Efficient parallelization of the multilevel fast multipole algorithm for the solution of large-scale scattering problems," IEEE Trans. Antennas Propag., vol. 56, no. 8, pp. 2335-2345, Aug. 2008.

[6] J. Fostier and F. Olyslager, "An asynchronous parallel MLFMA for scattering at multiple dielectric objects," IEEE Trans. Antennas Propag. vol. 56, no. 8, pp. 2346-2355, Aug. 2008.

[7] J. Fostier and F. Olyslager, "Provably scalable parallel multilevel fast multipole algorithm," Electron. Lett., vol. 44, no. 19, pp. 1111-1113, Sep. 2008.

[8] J. Fostier and F. Olyslager, "Full-wave electromagnetic scattering at extremely large 2-D objects," Electron. Lett., vol. 45, no. 5, pp. 245-246, Feb. 2009.

[9] Ö. Ergül and L. Gürel, "A hierarchical partitioning strategy for an efficient parallelization of the multilevel fast multipole algorithm," IEEE Trans. Antennas Propag., vol. 57, no. 6, pp. 1740-1750, Jun. 2009.
[10] J. M. Taboada, L. Landesa, F. Obelleiro, J. L. Rodriguez, J. M. Bertolo, M. G. Araujo, J. C. Mourino, and A. Gomez, "High scalability FMMFFT electromagnetic solver for supercomputer systems," IEEE Antennas Propag. Mag., vol. 51, no. 6, pp. 21-28, Dec. 2009.

[11] M. G. Araujo, J. M. Taboada, F. Obelleiro, J. M. Bertolo, L. Landesa, J. Rivero, and J. L. Rodriguez, "Supercomputer aware approach for the solution of challenging electromagnetic problems," vol. 101, pp. 241$256,2010$.

[12] J. M. Taboada, M. G. Araujo, J. M. Bertolo, L. Landesa, F. Obelleiro, and J. L. Rodriguez, "MLFMA-FFT parallel algorithm for the solution of large-scale problems in electromagnetics," Prog. Electromagn. Res. vol. 105, pp. 15-30, 2010.

[13] X.-M. Pan, W.-C. Pi, and X.-Q. Sheng, "On openmp parallelization of the multilevel fast multipole algorithm," Prog. Electromagn. Res., vol. 112, pp. 199-213, 2011.

[14] Ö. Ergül and L. Gürel, "Rigorous solutions of electromagnetics problems involving hundreds of millions of unknowns," IEEE Antennas Propag. Mag., vol. 53, no. 1, pp. 18-26, Feb. 2011.

[15] J. Song, C.-C. Lu, and W. C. Chew, "Multilevel fast multipole algorithm for electromagnetic scattering by large complex objects," IEEE Trans. Antennas Propag., vol. 45, no. 10, pp. 1488-1493, Oct. 1997.

[16] J. M. Taboada, L. Landesa, M. G. Araujo, J. M. Bertolo, F. Obelleiro, J. L. Rodriguez, J. Rivero, and G. Gajardo-Silva, "Supercomputer solutions of extremely large problems in electromagnetics: From ten million to one billion unknowns," in Proc. European Conf. on Antennas and Propagation (EuCAP), 2011, pp. 3221-3225.

[17] Ö. Ergül and L. Gürel, "Accuracy: the frequently overlooked parameter in the solution of extremely large problems," in Proc. European Conf. on Antennas and Propagation (EuCAP), 2011, pp. 3928-3931.

[18] Ö. Ergül and L. Gürel, "Benchmark solutions of large problems for evaluating accuracy and efficiency of electromagnetics solvers," in Proc. IEEE Antennas and Propagation Soc. Int. Symp., 2011.

[19] A. K. Dominek, M. C. Gilreath, and R. M. Wood, "Almond test body," United States Patent, no. 4809003, 1989.

[20] J. R. Mautz and R. F. Harrington, "H-field, E-field, and combined field solutions for conducting bodies of revolution," $A E \ddot{U}$, vol. 32, no. 4, pp. 157-164, Apr. 1978.

[21] S. M. Rao, D. R. Wilton, and A. W. Glisson, "Electromagnetic scattering by surfaces of arbitrary shape," IEEE Trans. Antennas Propag., vol. 30 no. 3, pp. 409-418, May 1982.

[22] H. van der Vorst, "Bi-CGSTAB: A fast and smoothly converging variant of Bi-CG for the solution of nonsymmetric linear systems," SIAM J. Sci. Stat. Comput., vol. 13, no. 2, pp. 631-644, Mar. 1992.

[23] L. Gürel, H. Bağcı, J. C. Castelli, A. Cheraly, and F. Tardivel, "Validation through comparison: measurement and calculation of the bistatic radar cross section (BRCS) of a stealth target," Radio Sci., vol. 38, no. 3, Jun. 2003 